\title{
Design of a Roll-Stabilized Mortar Projectile with Reciprocating Canards
}

\author{
Jonathan Rogers* and Mark Costello \\ Georgia Institute of Technology, Atlanta, Georgia 30332
}

DOI: $\underline{10.2514 / 1.47820}$

\begin{abstract}
The design of a canard-controlled mortar projectile using a bank-to-turn concept is presented. A unique feature of this smart mortar configuration is that it is equipped with a set of two reciprocating fixed-angle maneuver canards and a set of two reciprocating fixed-angle roll canards. An active control system is designed such that the roll canards set the body in the proper maneuver plane and the maneuver canards extend to perform trajectory corrections. Example results and Monte Carlo simulations demonstrate control system effectiveness in reducing dispersion error due to launch perturbations and winds. Comparison studies with a rolling airframe equipped with reciprocating maneuver canards show that the bank-to-turn approach offers more control authority, eliminates problematic angle-of-attack oscillations, and requires lower-bandwidth actuators.
\end{abstract}

\begin{tabular}{|c|c|}
\hline \multicolumn{2}{|r|}{ Nomenclature } \\
\hline$C_{L}, C_{D}$ & $=$ canard lift and drag coefficients \\
\hline$C_{i}$ & $=$ projectile aerodynamic coefficients \\
\hline$c_{\text {can }}$ & $=$ canard chord \\
\hline$D$ & $=$ projectile characteristic length \\
\hline$d_{\text {can }}$ & exposed canard span \\
\hline$e_{x}^{I}, e_{y}^{I}, e_{z}^{I}$ & $=$ position errors in the inertial reference frame \\
\hline$e_{y}^{N R}, e_{z}^{N R}$ & position errors in the no-roll reference frame \\
\hline$e_{x}^{P}, e_{y}^{P}, e_{z}^{P}$ & position errors in the projectile reference frame \\
\hline & mass moment-of-inertia matrix \\
\hline$K_{P p}, K_{\phi}$ & $=$ roll-rate and roll-angle gains \\
\hline $\begin{array}{l}K_{P \theta}, K_{D \theta}, \\
K_{I \theta}\end{array}$ & $\begin{array}{l}=\text { maneuver-canard proportional, derivative, and } \\
\text { integral gains }\end{array}$ \\
\hline$L, M, N$ & $\begin{aligned}= & \text { total external moments components on the } \\
& \text { projectile expressed in the body reference frame }\end{aligned}$ \\
\hline $\begin{array}{l}\mathbf{L}_{C_{i}}, \mathbf{D}_{C_{i}} \\
m\end{array}$ & $\begin{array}{l}=\text { lift and drag forces of the } i \text { th canard } \\
=\text { projectile mass }\end{array}$ \\
\hline$p, q, r$ & $\begin{array}{l}=\text { components of the angular velocity vector of } \\
\text { both the forward and aft bodies expressed in the } \\
\text { body reference frame }\end{array}$ \\
\hline & dynamic pressure at the projectile mass center \\
\hline$S_{\mathrm{can}_{M}}, S_{\mathrm{can}_{R}}$ & $=$ maneuver and roll-canard areas \\
\hline$u, v, w$ & $\begin{array}{l}=\text { translation velocity components of the center of } \\
\text { mass resolved in the body reference frame }\end{array}$ \\
\hline$V$ & magnitude of mass center velocity \\
\hline $\mathbf{V}_{C_{i}}$ & $\begin{array}{l}=\begin{array}{l}\text { local air velocity at the computation point of the } \\
i \text { th canard }\end{array}\end{array}$ \\
\hline$X, Y, Z$ & $\begin{aligned}= & \text { total external force components on the projectile } \\
& \text { expressed in the body reference frame }\end{aligned}$ \\
\hline$x, y, z$ & $\begin{array}{l}\text { position vector components of the center of mass } \\
\text { expressed in the inertial reference frame }\end{array}$ \\
\hline$\alpha$ & longitudinal aerodynamic angle of attack \\
\hline$\Delta \phi$ & roll-angle error signal \\
\hline & $=i$ th canard pitch angle \\
\hline & pitch-angle error signal \\
\hline & Euler roll, pitch, and yaw angles \\
\hline$\phi_{C_{i}}$ & $=i$ th canard azimuthal angle \\
\hline
\end{tabular}

Received 25 October 2009; revision received 28 January 2010; accepted for publication 8 February 2010 . Copyright $\odot 2010$ by the American Institute of Aeronautics and Astronautics, Inc. All rights reserved. Copies of this paper may be made for personal or internal use, on condition that the copier pay the $\$ 10.00$ per-copy fee to the Copyright Clearance Center, Inc., 222 Rosewood Drive, Danvers, MA 01923; include the code 0731-5090/10 and $\$ 10.00$ in correspondence with the CCC.

${ }^{*}$ Graduate Research Assistant, School of Aerospace Engineering. Member AIAA.

${ }^{\dagger}$ Sikorsky Associate Professor, School of Aerospace Engineering. Associate Fellow AIAA.

\section{Introduction}

D EVELOPMENT of accurate cost-efficient guided projectiles has presented weapons designers with numerous complex technical challenges over the past several decades. Guided projectiles, in contrast to guided missiles, must withstand extreme acceleration loads at launch and endure high spin rates. Control mechanisms and onboard electronics suites must be relatively small due to space limitations. Furthermore, guided projectiles must be relatively inexpensive, since they are often fired in large quantities. However, the ability to reduce dispersion error and provide a precision-strike capability using standard munitions has continually motivated smart weapons developers to overcome these technical challenges.

Control of flight vehicles, particularly projectiles, using canard mechanisms is not a new idea and has been employed extensively in the missile community and, more recently, in the smart weapons community. A large collection of aerodynamic data for numerous canard-equipped missile configurations has been amassed by the U.S. Air Force [1]. In the guided projectile community, several studies and development programs have considered use of canard mechanisms for flight control purposes onboard both fin- and spin-stabilized indirect fire munitions. Smith et al. [2] have explored the application of canard control to a spin-stabilized projectile for dispersion error reduction. Their design used a seekerbased guidance system and mounted canards on a rolling bearing spinning slower than the body in order to reduce canard actuator power and bandwidth. Later, Costello [3] investigated the use of canards onboard an artillery shell for the purpose of range extension. Example results and trade studies showed that dramatic range increases could be accomplished using reasonably sized canards.

Numerous guided artillery projectiles developed over the past three decades have employed canard control mechanisms. One of the first such development programs was Copperhead [4,5], a $155 \mathrm{~mm}$ artillery round that used four dithering rear tail fins for roll stabilization and maneuver control as well as four fixed canards placed forward on the body for maneuver augmentation. Another program, the Low Cost Competent Munition development effort conducted jointly by the U.S. Army and Navy [6], explored the use of extendable fixed canards for use as drag brakes as well as dithering canards for use in precise trajectory corrections. The U.S. Navy's Extended-Range Guided Munition [7,8] combined use of a rocket motor, tail fins, and forward-placed canards to significantly extend the range of a 5 in. projectile for naval guns. Most recently, the Army's Excalibur [9] and Precision Guidance Kit [10] development programs for $155 \mathrm{~mm}$ projectiles have demonstrated the feasibility of using canard mechanisms to successfully guide cannon-launched munitions. 
The work reported here describes the implementation of a bank-toturn control system on a standard mortar projectile. The control system uses two sets of canards: a pair of roll-stabilization canards and a pair of maneuver canards. This design is unique in that all canards are fixed-angle and can extend and retract from the body as required (termed reciprocating) in order to generate desired control forces, as shown in Fig. 1. The roll-stabilization canards regulate projectile spin rate and set the projectile to the desired roll angle, and the maneuver canards are responsible for trajectory corrections. Specifically, the control system is designed to track an input ballistic trajectory, reducing dispersion error due to launch disturbances and winds. The paper begins with a description of the projectile and canard dynamic models as well as a short description of the advantages of the proposed design. Then, example cases show control system performance and control authority predictions for the example projectile. Monte Carlo simulations incorporating sensor errors, atmospheric winds, and launch errors demonstrate that the control system is effective at significantly reducing dispersion error. All cases are compared with an equivalent rolling configuration to demonstrate the advantages of roll-stabilization from a basic flight control perspective. A final study examines the effect of maneuvercanard placement on the body.

\section{Dynamic Model}

This section describes the projectile dynamic model as well as the methodology used to compute forces and moments on the projectile body. Note that throughout this paper the following shorthand notation is used for trigonometric angles: $s_{\alpha}=\sin (\alpha), c_{\alpha}=\cos (\alpha)$, and $t_{\alpha}=\tan (\alpha)$.

\section{A. Projectile Dynamic Model}

Projectile motion is described by a rigid body six-degree-offreedom model. A ground-based frame is used as the inertial reference frame $I$, and the body-fixed reference frame $P$ is defined using the conventional aerospace Euler rotation sequence [11,12]. Three translational kinematic equations, relating derivatives of position states to velocity states, are given by

$$
\left\{\begin{array}{c}
\dot{x} \\
\dot{y} \\
\dot{z}
\end{array}\right\}=\left[\begin{array}{ccc}
c_{\theta} c_{\psi} & s_{\phi} s_{\theta} c_{\psi}-c_{\phi} s_{\psi} & c_{\phi} s_{\theta} c_{\psi}+s_{\phi} s_{\psi} \\
c_{\theta} s_{\psi} & s_{\phi} s_{\theta} s_{\psi}+c_{\phi} c_{\psi} & c_{\phi} s_{\theta} s_{\psi}-s_{\phi} c_{\psi} \\
-s_{\theta} & s_{\phi} c_{\theta} & c_{\phi} c_{\theta}
\end{array}\right]\left\{\begin{array}{c}
u \\
v \\
w
\end{array}\right\}
$$

The rotational kinematic equations relate derivatives of Euler angles to angular velocity states and are given by

$$
\left\{\begin{array}{c}
\dot{\phi} \\
\dot{\theta} \\
\dot{\psi}
\end{array}\right\}=\left[\begin{array}{ccc}
1 & s_{\phi} t_{\theta} & c_{\phi} t_{\theta} \\
0 & c_{\phi} & -s_{\phi} \\
0 & s_{\phi} / c_{\theta} & c_{\phi} / c_{\theta}
\end{array}\right]\left\{\begin{array}{l}
p \\
q \\
r
\end{array}\right\}
$$

Three translational dynamic equations are obtained using a force balance equation on the projectile written in the body frame, given by

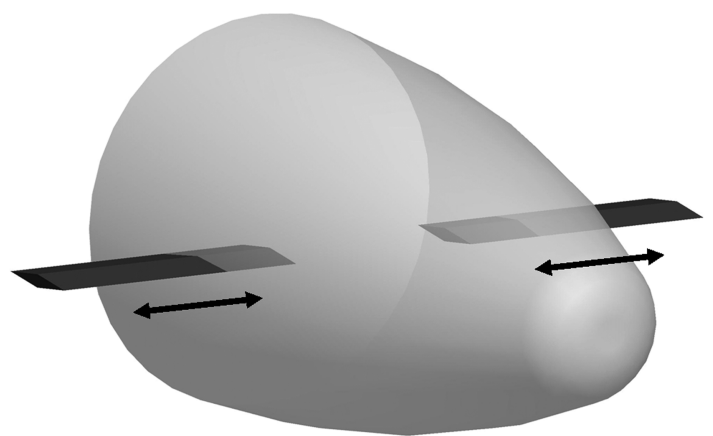

Fig. 1 Diagram of reciprocating canard motion.

$$
\left\{\begin{array}{c}
\dot{u} \\
\dot{v} \\
\dot{w}
\end{array}\right\}=\frac{1}{m}\left\{\begin{array}{c}
X \\
Y \\
Z
\end{array}\right\}-\left[\begin{array}{ccc}
0 & -r & q \\
r & 0 & -p \\
-q & p & 0
\end{array}\right]\left\{\begin{array}{c}
u \\
v \\
w
\end{array}\right\}
$$

Note that in Eq. (3) the terms $X, Y$, and $Z$ are the sum of weight, aerodynamic, and canard forces resolved in the projectile reference frame. The rotational dynamic equations are obtained by writing a moment equation about the projectile mass center. Resolved into the projectile reference frame, they are given by

$$
\left\{\begin{array}{c}
\dot{p} \\
\dot{q} \\
\dot{r}
\end{array}\right\}=[I]^{-1}\left[\left\{\begin{array}{c}
L \\
M \\
N
\end{array}\right\}-\left[\begin{array}{ccc}
0 & -r & q \\
r & 0 & -p \\
-q & p & 0
\end{array}\right][I]\left\{\begin{array}{c}
p \\
q \\
r
\end{array}\right\}\right]
$$

These 12 equations are integrated forward in time using a fourthorder Runge-Kutta algorithm to obtain a single trajectory. Furthermore, the flight simulation using the mathematical model described above has been validated against spark range data for a $25 \mathrm{~mm}$ fin-stabilized projectile, showing excellent agreement between predicted and experimental data [13].

\section{B. Forces and Moments}

Projectile motion is driven by weight and applied aerodynamic loads. Aerodynamic forces and moments are calculated within the flight simulation using the PRODAS aerodynamic expansion [14]. The total forces on the projectile can be expressed as

$$
\left\{\begin{array}{c}
X \\
Y \\
Z
\end{array}\right\}=\left\{\begin{array}{c}
X_{W} \\
Y_{W} \\
Z_{W}
\end{array}\right\}+\left\{\begin{array}{c}
X_{A} \\
Y_{A} \\
Z_{A}
\end{array}\right\}+\left\{\begin{array}{c}
X_{M} \\
Y_{M} \\
Z_{M}
\end{array}\right\}+\left\{\begin{array}{c}
X_{C} \\
Y_{C} \\
Z_{C}
\end{array}\right\}
$$

Note that in Eq. (5) the subscripts $W, A, M$, and $C$ denote weight, aerodynamic, Magnus, and canard forces, respectively. The weight force is given by

$$
\left\{\begin{array}{l}
X_{W} \\
Y_{W} \\
Z_{W}
\end{array}\right\}=m_{P} g\left\{\begin{array}{c}
-s_{\theta} \\
s_{\phi} c_{\theta} \\
c_{\phi} c_{\theta}
\end{array}\right\}
$$

Components of the aerodynamic and Magnus forces are calculated using the following expressions. Note that the Magnus force acts at the Magnus center of pressure, which is different from the center of pressure of the steady aerodynamic forces:

$$
\begin{gathered}
X_{A}=-\frac{\pi}{8} \rho V^{2} D^{2}\left(C_{X 0}+C_{X 2} \varepsilon^{2}\right) \\
Y_{A}=-\frac{\pi}{8} \rho V^{2} D^{2}\left[C_{N A} \frac{v}{V}\right] \\
Z_{A}=-\frac{\pi}{8} \rho V^{2} D^{2}\left[C_{N A} \frac{w}{V}\right] \\
X_{M}=0 \\
Y_{M}=\frac{\pi}{8} \rho V^{2} D^{2} \frac{p D}{2 V}\left[C_{Y P A} \frac{w}{V}\right] \\
Z_{M}=-\frac{\pi}{8} \rho V^{2} D^{2} \frac{p D}{2 V}\left[C_{Y P A} \frac{v}{V}\right]
\end{gathered}
$$

Equation (7) uses the following expression for calculation of total angle of attack: 


$$
\varepsilon=\frac{\sqrt{v^{2}+w^{2}}}{\sqrt{u^{2}+v^{2}+w^{2}}}
$$

Canard forces will be discussed in the next section. The total applied body moments contain steady, unsteady, canard, and Magnus terms denoted by subscripts $S, U, C$, and $M$, respectively:

$$
\left\{\begin{array}{c}
L \\
M \\
N
\end{array}\right\}=\left\{\begin{array}{c}
L_{S} \\
M_{S} \\
N_{S}
\end{array}\right\}+\left\{\begin{array}{c}
L_{U} \\
M_{U} \\
N_{U}
\end{array}\right\}+\left\{\begin{array}{c}
L_{M} \\
M_{M} \\
N_{M}
\end{array}\right\}+\left\{\begin{array}{c}
L_{C} \\
M_{C} \\
N_{C}
\end{array}\right\}
$$

The steady body aerodynamic moment is computed with a cross product between the distance vector from the center of gravity to the center of pressure and the steady body aerodynamic force vector above. Likewise, the Magnus aerodynamic moment is computed with a cross product between the distance vector from the center of mass to the center of Magnus force and the Magnus force vector. For each canard, the canard aerodynamic moment is computed with a cross product between the distance vector from the center of mass to the canard computation point and the canard force vector. The canard moment is then summed over all canards and input into Eq. (14).

The unsteady body aerodynamic moment provides a damping source for projectile angular motion. Expansions for the unsteady aerodynamic moment are given by

$$
\begin{gathered}
L_{U}=\frac{\pi}{8} \rho V^{2} D^{3}\left[C_{L D D}+\frac{p D}{2 V} C_{L P}\right] \\
M_{U}=\frac{\pi}{8} \rho V^{2} D^{3}\left[\frac{q D}{2 V} C_{M Q}\right] \\
N_{U}=\frac{\pi}{8} \rho V^{2} D^{3}\left[\frac{r D}{2 V} C_{M Q}\right]
\end{gathered}
$$

The aerodynamic coefficients and aerodynamic center distances are all a function of local Mach number at the center of mass of the projectile. Computationally, these Mach-number-dependent parameters are obtained by a table lookup scheme using linear interpolation.

\section{Canard Aerodynamic Model}

The aerodynamic force due to a single canard is modeled as a point force acting at the canard aerodynamic center of pressure. The $i$ th canard orientation is defined by two angles: canard azimuthal angle $\phi_{C_{i}}$ and pitch angle $\delta_{C_{i}}$. In this study, both angles are fixed throughout flight. The canard force exerted by the $i$ th canard is therefore

$$
\left\{\begin{array}{c}
X_{C_{i}} \\
Y_{C_{i}} \\
Z_{C_{i}}
\end{array}\right\}=q_{C_{i}} S_{i}\left[T_{C_{i}}\right]\left\{\begin{array}{c}
C_{L} s_{\alpha_{C i}-\delta_{C i}}-C_{D} c_{\alpha_{C i}-\delta_{C i}} \\
0 \\
-C_{L} c_{\alpha_{C i}-\delta_{C i}}-C_{D} s_{\alpha_{C i}-\delta_{C i}}
\end{array}\right\}
$$

where $q_{C_{i}}$ is the dynamic pressure at the canard computation point, $S_{i}$ is the canard reference area, and $T_{C_{i}}$ is the transformation from the local canard reference frame to the projectile body frame. Canard lift and drag coefficients are functions of total canard angle of attack and local Mach number, according to

$$
\begin{gathered}
C_{L}=C_{L 1} \alpha_{C_{i}}+C_{L 3} \alpha_{C_{i}}^{3}+C_{L 5} \alpha_{C_{i}}^{5} \\
C_{D}=C_{D 0}+C_{D 2} \alpha_{C_{i}}^{2}+C_{I} C_{L}^{2}
\end{gathered}
$$

All coefficients in Eqs. (19) and (20) are Mach-number-dependent. The total canard angle of attack $\alpha_{C_{i}}$ is given by

$$
\alpha_{C_{i}}=\tan ^{-1}\left(\frac{w_{C_{i}}}{u_{C_{i}}}\right)
$$

where $w_{C_{i}}$ and $u_{C_{i}}$ are components of the local velocity vector at the canard computation point. A diagram showing canard angles, canard drag force, and canard lift force is shown in Fig. $\underline{2}$.

\section{Control System Design}

The flight control system used to perform trajectory corrections computes required canard area for the roll-stabilization and maneuver canards. Therefore, there are two separate control loops: one for regulating projectile spin rate to zero and setting the airframe in the desired roll orientation, and the other for tracking a command trajectory using the maneuver-canard set. The roll controller uses proportional-derivative control, and the maneuver controller uses proportional-integral-derivative (PID) control. These standard and straightforward flight control techniques are chosen because the purpose of this study is to examine the performance of the control mechanism. It is therefore noted that although the roll and maneuver control laws may not represent the optimal control scheme for the proposed system, they are sufficient to demonstrate basic control mechanism performance. Note that throughout this section and the Results section, the equivalent terms roll-stabilization and bank-toturn are used interchangeably.

First, a lead distance is added to current projectile range to produce a lead range value $\left(x_{\text {com }}\right)$ used to index the command trajectory table. This table contains a set of discrete points describing the commanded trajectory. Interpolating this table using $x_{\text {com }}$, the desired projectile location can be obtained. Then, a position error vector in the inertial frame is calculated according to

$$
\left\{\begin{array}{l}
e_{x}^{I} \\
e_{y}^{I} \\
e_{z}^{I}
\end{array}\right\}=\left\{\begin{array}{l}
x_{\mathrm{com}} \\
y_{\mathrm{com}} \\
z_{\mathrm{com}}
\end{array}\right\}-\left\{\begin{array}{l}
x \\
y \\
z
\end{array}\right\}
$$

where the subscript com denotes the commanded positions. This error vector is transformed into the projectile reference frame according to

$$
\left\{\begin{array}{c}
e_{x}^{P} \\
e_{y}^{P} \\
e_{z}^{P}
\end{array}\right\}=\left[\begin{array}{ccc}
c_{\theta} c_{\psi} & c_{\theta} s_{\psi} & -s_{\theta} \\
s_{\phi} s_{\theta} c_{\psi}-c_{\phi} s_{\psi} & s_{\phi} s_{\theta} s_{\psi}+c_{\phi} c_{\psi} & s_{\phi} c_{\theta} \\
c_{\phi} s_{\theta} c_{\psi}+s_{\phi} s_{\psi} & c_{\phi} s_{\theta} s_{\psi}-s_{\phi} c_{\psi} & c_{\phi} c_{\theta}
\end{array}\right]\left\{\begin{array}{c}
e_{x}^{I} \\
e_{y}^{I} \\
e_{z}^{I}
\end{array}\right\}
$$

The roll-stabilization and maneuver controllers use this body-frame error vector to compute required canard areas.

\section{A. Roll Control System}

The roll-stabilization canards are located on each side of the projectile and have opposing pitch angles. The moment generated by this fixed-angle configuration counteracts the roll torque exerted by the fin set at the rear of the projectile. By varying roll-canard area, a roll torque is introduced. The roll control system first transforms the $y$ and $z$ components of the body-frame error vector into the no-roll reference frame (denoted by the superscript NR):

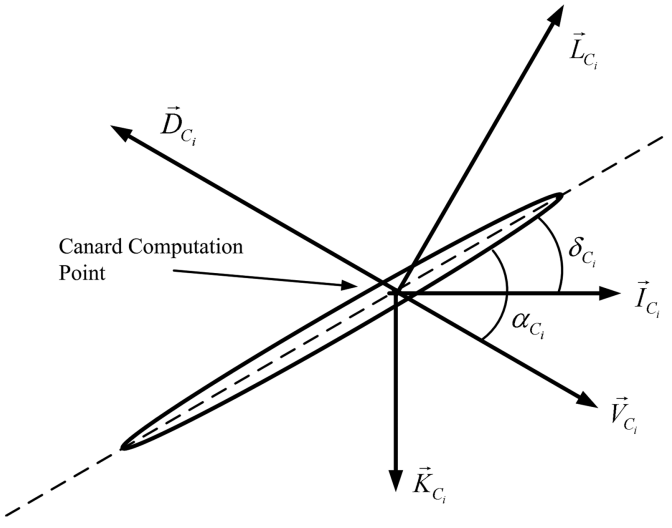

Fig. 2 Diagram of canard angles and forces. 


$$
\left\{\begin{array}{l}
e_{y}^{\mathrm{NR}} \\
e_{z}^{\mathrm{NR}}
\end{array}\right\}=\left\{\begin{array}{l}
s_{\phi} e_{y}^{P}+c_{\phi} e_{z}^{P} \\
c_{\phi} e_{y}^{P}-s_{\phi} e_{z}^{P}
\end{array}\right\}
$$

Then, the desired roll angle is computed according to

$$
\phi=\tan ^{-1}\left(\frac{e_{z}^{\mathrm{NR}}}{e_{y}^{\mathrm{NR}}}\right)+\frac{\pi}{2}
$$

Defining the roll-angle error signal by simply subtracting the roll angle from the desired roll angle can lead to wrapping problems. To avoid this, the roll-angle error signal $\Delta \phi$ is computed using the expression

$$
\Delta \phi=\tan ^{-1}\left(\frac{s_{\phi} c_{\phi D}-c_{\phi} s_{\phi D}}{c_{\phi} c_{\phi D}+s_{\phi} s_{\phi D}}\right)
$$

Finally, roll-stabilization canard area is computed according to

$$
S_{\mathrm{can}_{R}}=K_{P p} p+K_{\phi} \Delta \phi
$$

where $K_{P_{p}}$ and $K_{\phi}$ are user-defined gains. Although proportionalderivative controllers of the type shown in Eq. (27) are susceptible to some steady-state error, experience with this roll controller have shown that with the use of reasonable gains, such errors are negligible and do not noticeably impact overall performance. The computed roll-canard area is limited such that canard area can never be negative and can never exceed the maximum roll-canard area. In addition, deadbands are implemented within the roll control system to deactivate roll-angle tracking when error signals are sufficiently small.

\section{B. Maneuver Control System}

The maneuver canards are located on each side of the body (but orthogonal to the roll canards) and have identical pitch angles. Therefore, by varying canard area, the maneuver control mechanism can exert a variable pitching moment on the projectile for control purposes. The maneuver control system computes a pitch-angle error signal $\theta_{e}$ using the expression

$$
\theta_{e}=\tan ^{-1}\left(\frac{w}{u}\right)-\tan ^{-1}\left(\frac{e_{z}^{P}}{e_{x}^{P}}\right)
$$

Note that this definition properly accounts for angle of attack and atmospheric winds in the definition of the pitch-angle error. A PID control then attempts to regulate this error to zero according to

$$
S_{\operatorname{can}_{M}}=K_{P \theta} \theta_{e}+K_{D \theta} \dot{\theta}_{e}+K_{I \theta} \int_{0}^{t} \theta_{e} \mathrm{~d} t
$$

This computed canard area is also limited such that canard area can never be negative and can never exceed the maximum canard area.

Note that while the roll and maneuver control systems described above may be largely decoupled, maneuver-canard placement trade study results shown below demonstrate that the responses of the two control systems are dependent on one another, and therefore design of the roll and maneuver control loops cannot be performed completely independently.

\section{Advantages of Proposed Design}

Numerous smart weapons development programs have used canard control mechanisms. Canard mechanisms typically exert control force through variation of canard pitch angle $\delta_{c}$ (dithering canards) or through variation of exposed fixed-angle canard area $S_{\text {can }}$ (reciprocating canards). Furthermore, canard control is applied to control either a rolling round by modulating canard force at the projectile roll rate or a roll-stabilized round by using canards to despin the projectile and exert maneuver control force. The design proposed here possesses several key advantages over all of these designs.

Diagrams of reciprocating canard motion are shown in Figs. 1 and $\underline{3}$ and differ from dithering canards in their method of control.
Side View of Reciprocating Canard on Right Side of Body

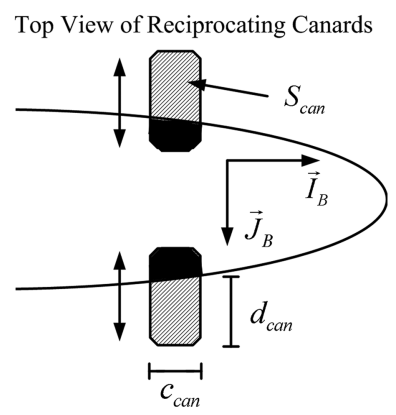

Fig. 3 Schematic of reciprocating canard design.

Note that reciprocating designs vary exposed canard area while maintaining constant deflection angle, and dithering designs vary deflection angle while maintaining constant exposed area. Although four canards are used for the proposed design, a set of two canards is shown in Figs. 1 and 3 for simplicity. One advantage of reciprocating canard designs is the capability to retract the canards inside the body when not in use, reducing drag penalties when no control is required. This can be especially important in instances in which the designer wishes to maximize range and impact velocity. Furthermore, dithering canard actuators must overcome a torque caused by an offset between the actuator hinge and the canard aerodynamic center of pressure. This actuator moment can be quite substantial, especially at higher flight velocities, and can lead to large actuators requiring more power. Note that the canard center of pressure varies along the canard chord with Mach number, meaning that the actuator hinge point can never lie at the center of pressure for all flight regimes. Use of reciprocating canards can significantly reduce this burden on actuators, since use of low-friction roller bearings require very low power. Constant extension of the canards would not require any power, unlike dithering designs in which moments exerted on the canard actuator must be continuously overcome during constant deflection.

Roll-stabilized designs possess several advantages compared with rolling designs. First, as shown in the Results section, the ability of the roll-stabilized design to exert control continuously rather than through only a portion of the roll cycle significantly increases control efficiency and overall maneuver authority. In addition, rolling designs often require high-bandwidth actuators to modulate canard deflection or canard extension at the projectile roll rate. This can lead to complex or expensive actuators and large onboard power demands. Roll-stabilized designs, however, do not require highfrequency actuator motion and can use inexpensive mechanisms requiring relatively little power.

The design proposed here is unique in that it combines the advantages of reciprocating canards with the benefits of rollstabilization. The result is a system that possesses several key advantages over all other canard designs that can be applied successfully to many different classes of munitions.

\section{Results}

The example mortar round used for all studies in this paper is a typical mortar projectile with mass, axial moment of inertia, and transverse moment of inertia given by $4.6 \mathrm{~kg}, 0.0037 \mathrm{~kg}-\mathrm{m}^{2}$, and $0.0500 \mathrm{~kg}-\mathrm{m}^{2}$, respectively. Total projectile length is approximately $0.530 \mathrm{~m}$, and mass center station-line position referenced from the aft end of the round is approximately $0.28 \mathrm{~m}$. Except where specified, all canards are placed relatively close to the projectile c.g., approximately $2.8 \mathrm{~cm}$ in front. Figure 4 shows a drawing of the canard-equipped mortar round, showing canards both partially retracted and fully extended. Note that the roll-stabilization and maneuver-canard sets are orthogonal to one another.

Throughout this section, performance of the roll-stabilized projectile will be compared with an equivalent rolling projectile (referred to as rolling). This rolling projectile has an identical configuration to the roll-stabilized projectile, except that the 
Canards Retracted

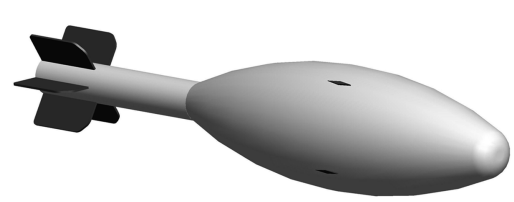

Fig. 4 Example mortar projectile equipped with canards in retracted and deployed configuration.
Canards Extended

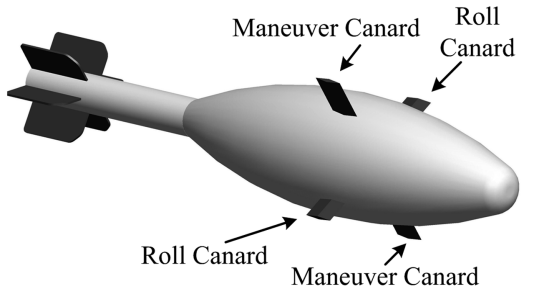

roll-stabilization control is deactivated and all roll-stabilization canard areas are set to zero.

\section{A. Example Trajectory}

An example trajectory is described to demonstrate flight control system performance as well as to compare general performance trends with the rolling projectile. A ballistic case using unperturbed initial conditions is used as the command trajectory. Perturbed initial conditions are then used to demonstrate control performance. All initial conditions are shown in Table 1 , and control system parameters are shown in Table 2 . Note that in Table 2 , total canard area includes both canards in a given set. Figures 5-11 show the results of the example trajectory. First, note that control activation occurs at apogee in order to reduce drag penalties early in flight. Figures 5-7 demonstrate that both the roll-stabilized and rolling controlled trajectories are successful in reducing dispersion error, recording controlled miss distances of less than $4 \mathrm{~m}$, compared with a total miss distance of approximately $80 \mathrm{~m}$ for the ballistic case. Figure 8 , showing roll-rate profiles, demonstrates the slight increase in roll damping that occurs during canard extension. Note that the roll-stabilization case successfully despins the projectile when control is applied. Slight roll-rate perturbations occur thereafter as the body is adjusted to the proper roll orientation. Figure 9 demonstrates that relatively small angles of attack are induced due to the placement of canards near the mass center. However, note the high-frequency angle-of-attack oscillations that occur in the rolling case as canards reciprocate at the roll frequency. This contrasts with the smooth angle of attack of the roll-stabilized case. Figure 10 demonstrates how roll canards are applied throughout flight for the roll-stabilized case, adjusting roll angle while counteracting the roll torque due to the rear fin set. Note that all three disturbances occur due to reversal or predicted reversal of the deflection error and that the roll-canard area required to regulate spin rate to zero changes

Table 1 Initial conditions for example simulation

\begin{tabular}{lccccc}
\hline \hline State & Unperturbed & Perturbed & State & Unperturbed & Perturbed \\
\hline$x, \mathrm{~m}$ & 0.0 & 0.0 & $u, \mathrm{~m} / \mathrm{s}$ & 294.9 & 292.8 \\
$y, \mathrm{~m}$ & 0.0 & 0.0 & $v, \mathrm{~m} / \mathrm{s}$ & 0.0 & 1.22 \\
$z, \mathrm{~m}$ & 0.0 & 0.0 & $w, \mathrm{~m} / \mathrm{s}$ & 0.0 & -1.83 \\
$\phi, \mathrm{deg}$ & 0.0 & 0.0 & $p, \mathrm{rad} / \mathrm{s}$ & 0.0 & 0.0 \\
$\theta, \mathrm{deg}$ & 51.57 & 51.68 & $q, \mathrm{rad} / \mathrm{s}$ & 0.0 & 1.0 \\
$\psi, \mathrm{deg}$ & 0.0 & -0.172 & $r, \mathrm{rad} / \mathrm{s}$ & 0.0 & 1.3 \\
\hline \hline
\end{tabular}

Table 2 Control system parameters

\begin{tabular}{lc}
\hline \hline Lead distance & $244 \mathrm{~m}$ \\
Roll angle gain $K_{\phi}$ & 0.08 \\
Roll rate gain $K_{P p}$ & 0.01 \\
Maneuver proportional gain $K_{P \theta}$ & 1.6 \\
Maneuver derivative gain $K_{D \theta}$ & 0.001 \\
Maneuver integral gain $K_{I \theta}$ & $5 \times 10^{-4}$ \\
Total roll-canard area & $5.57 \mathrm{~cm}^{2}$ \\
Total maneuver-canard area & $16.6 \mathrm{~cm}^{2}$ \\
Roll-canard deflection angle & $15.0 \mathrm{deg}$ \\
Maneuver-canard deflection angle & $4.27 \mathrm{deg}$ \\
\hline \hline
\end{tabular}

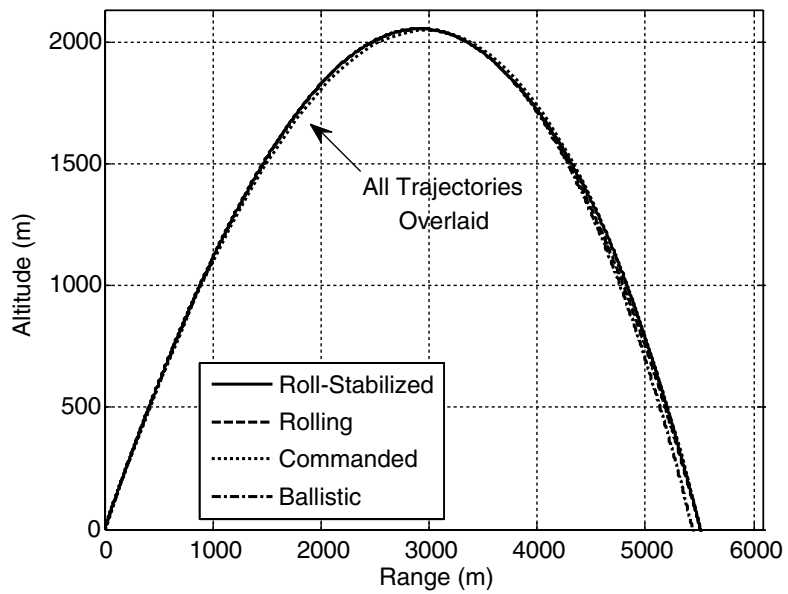

Fig. 5 Altitude vs range.

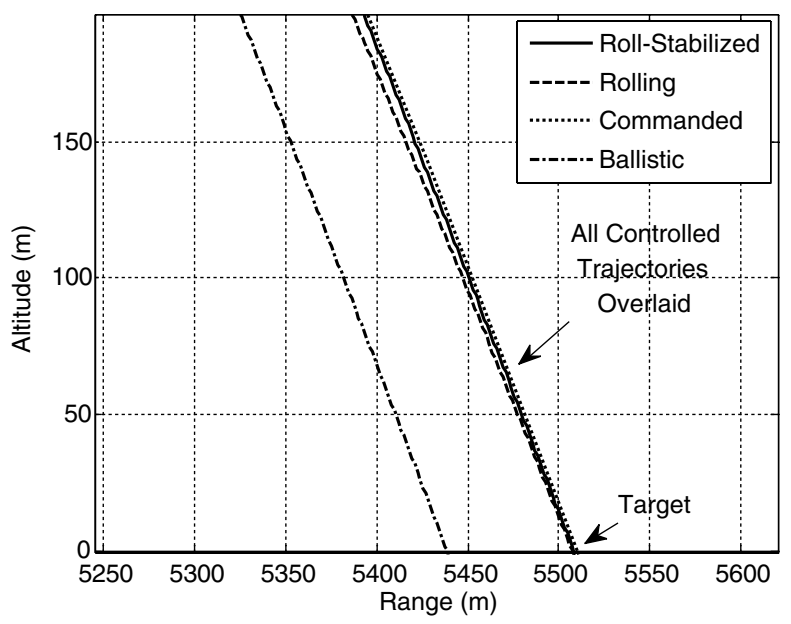

Fig. 6 Zoom view of target area; altitude vs range.

gradually as speed increases during the terminal portion of the trajectory. Finally, Fig. 11 demonstrates the smooth application of maneuver canards for the roll-stabilized case, contrasted with the oscillatory application of maneuver canards for the rolling case. Although not shown here, total velocity-time histories for all cases varied only on the order of a $1 \%$, demonstrating that the drag penalty associated with canards of this size is relatively small.

This example case highlights several key advantages of the bankto-turn system. First, extension and retraction of the canards at the projectile roll rate induces significant angle-of-attack oscillations that can be highly detrimental to the performance of sensor suites and state estimators. These oscillations occur as well in roll rate due to oscillatory roll damping. Furthermore, canard reciprocation at the roll frequency requires fast actuator response times (on the order of 10-20 Hz), demonstrated by high-frequency motion of the maneuver canards in Fig. 11. In contrast, the roll-stabilization system exhibits 


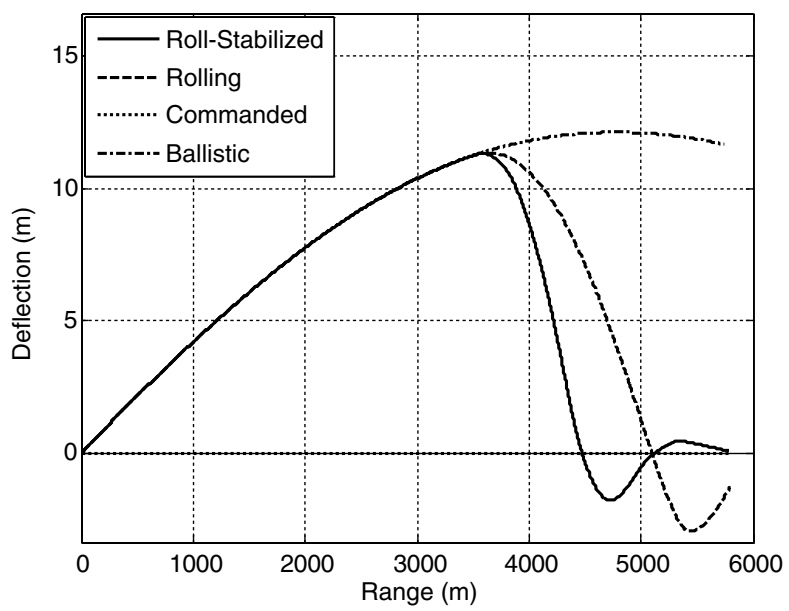

Fig. 7 Deflection vs range.

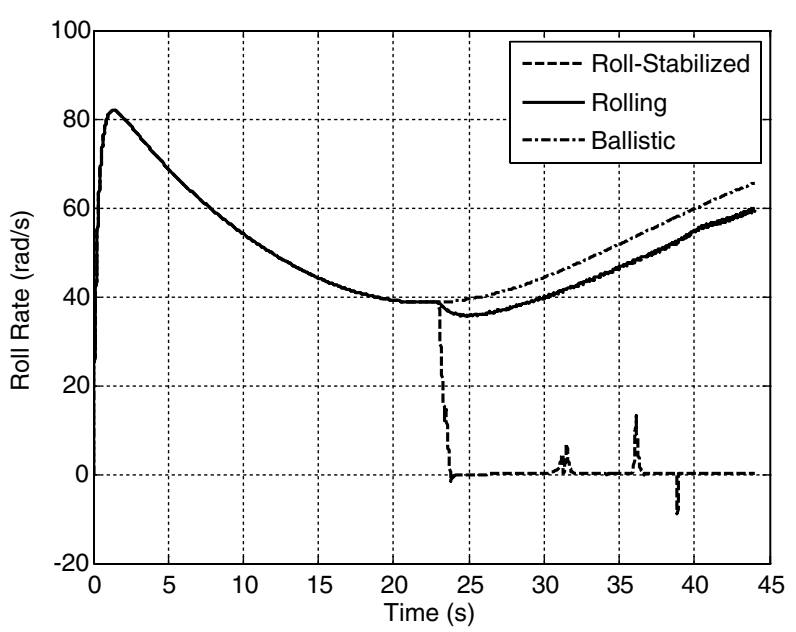

Fig. 8 Roll rate vs time.
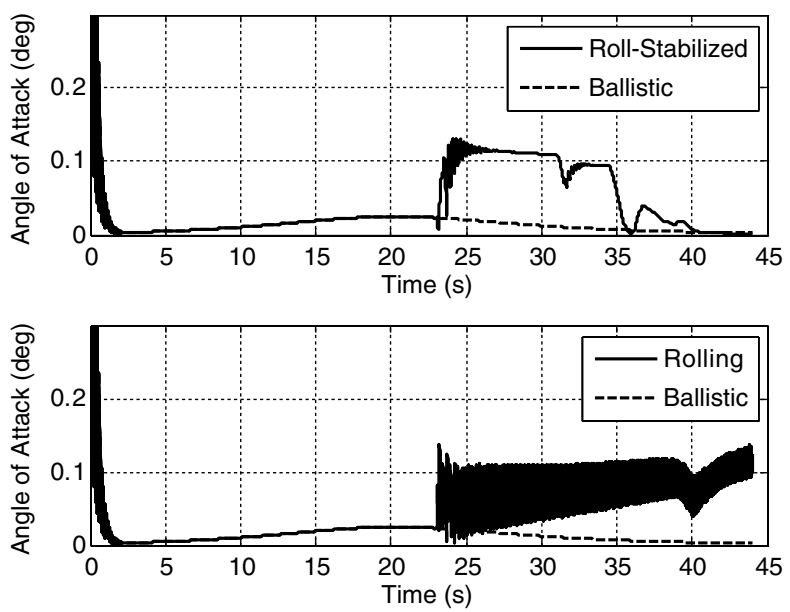

Fig. 9 Angle of attack vs time.

smooth angle of attack and roll-rate response while allowing lowbandwidth actuators to be used. Finally, use of reciprocating canards as opposed to typical variable deflection angle designs provides the ability to retract canards into the body when not in use, significantly reducing drag penalties due to canard deployment.

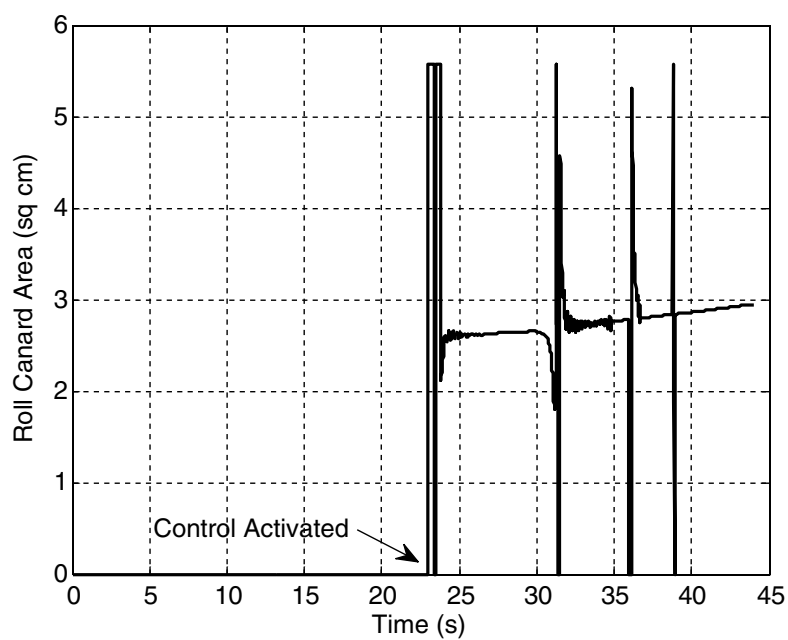

Fig. 10 Roll-canard area-time history for roll-stabilized case.
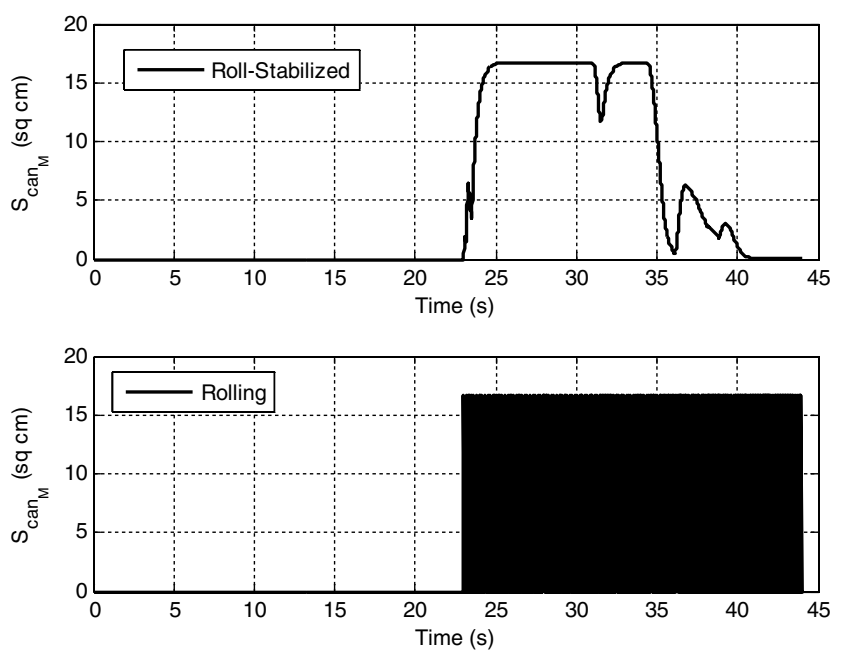

Fig. 11 Maneuver-canard area-time histories.

\section{B. Control Authority Study}

Another study is presented that explores the control authority of the roll-stabilized projectile in comparison with the rolling projectile. In this case, control is applied at apogee to simply deflect the round in the positive inertial $y$ direction (i.e., rightward-looking downrange). Because control is saturated throughout the controlled portion of the trajectory, this technique yields a good estimate of the maximum maneuver authority of the system. Figure 12 shows that the

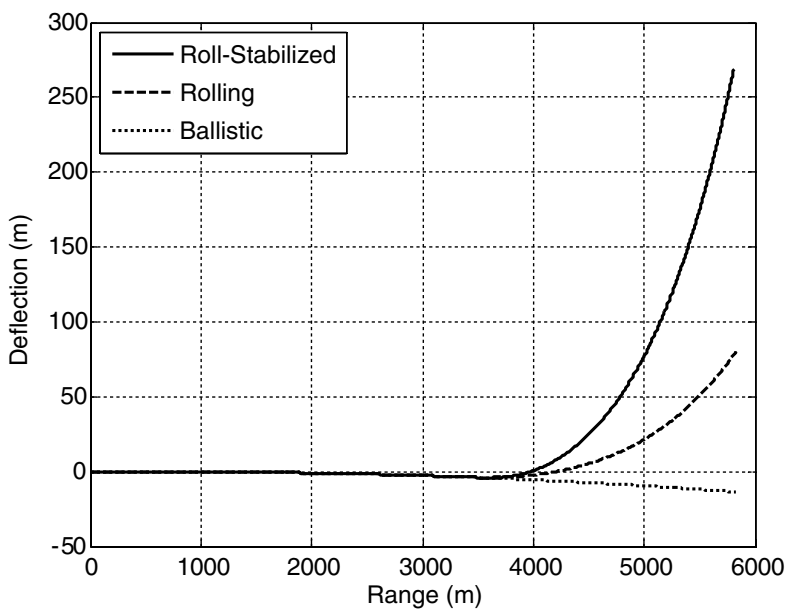

Fig. 12 Deflection vs range. 

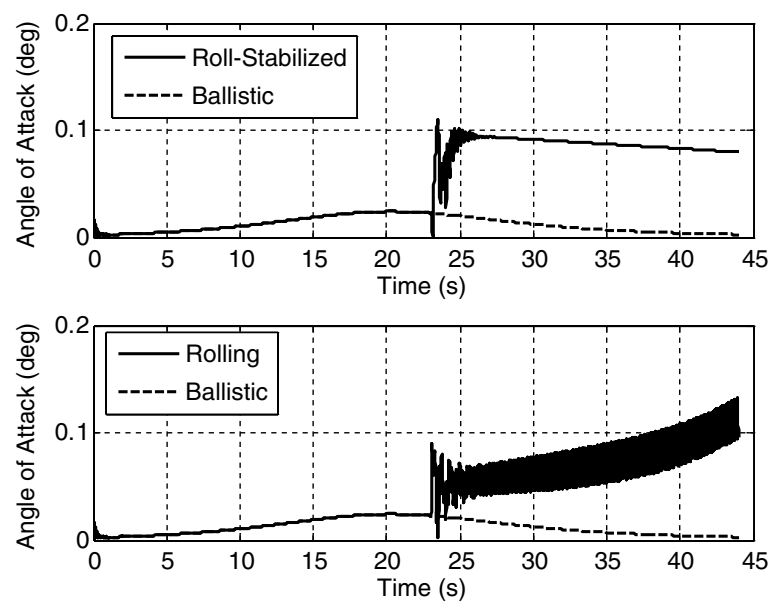

Fig. 13 Angle of attack vs time.
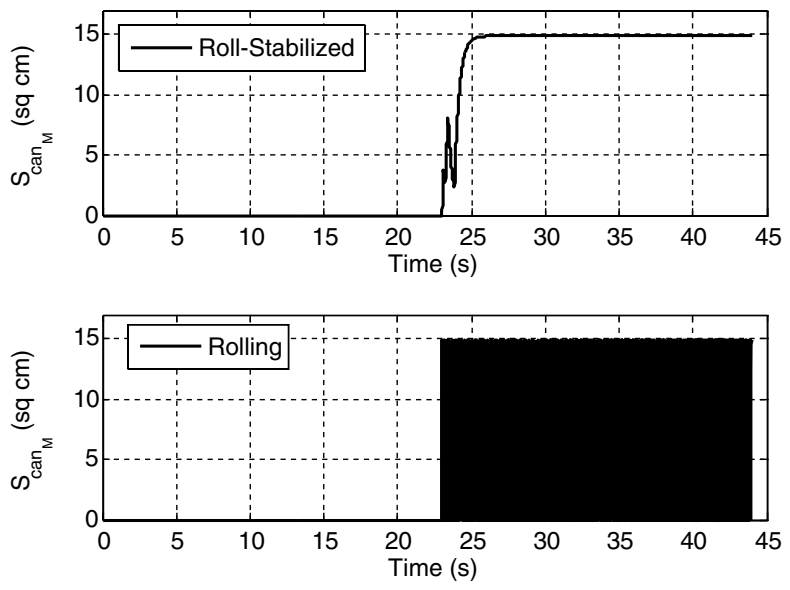

Fig. 14 Maneuver-canard area-time histories.

roll-stabilized configuration is able to achieve a maximum deflection of roughly $275 \mathrm{~m}$ compared with the ballistic case. This is three to four times the deflection achieved by the rolling case, which showed approximately $85 \mathrm{~m}$ deflection compared with the ballistic case. Figure 13 explains this maneuver authority advantage by demonstrating that the roll-stabilized round can induce angle of attack continuously, whereas the rolling projectile can only exert control through a portion of the roll cycle. Therefore, the bank-toturn configuration can be expected to exhibit significantly more maneuver authority than rolling configurations. Figure 14 shows maneuver-canard area-time histories for this case. Roll-canard time histories, omitted for brevity, show that the roll canards extend smoothly and show relatively constant area, fixing the airframe in the optimal roll orientation.
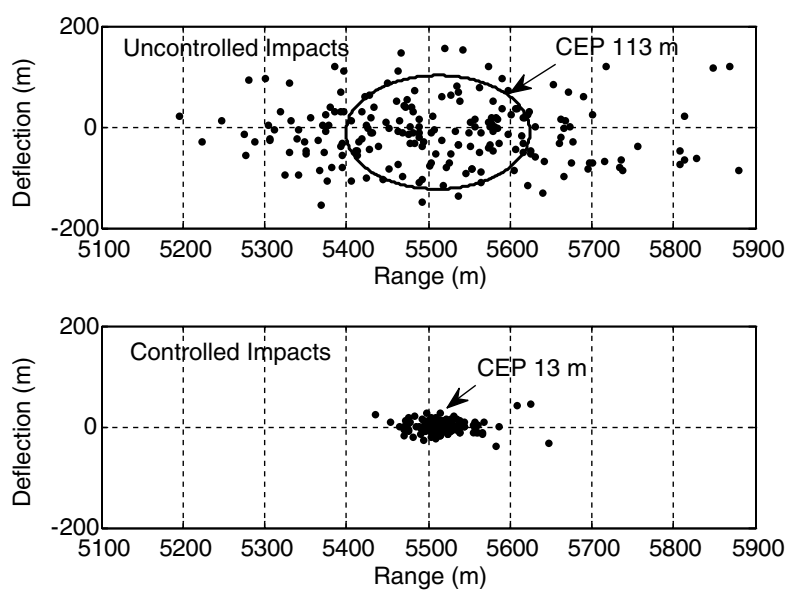

Fig. 15 Example dispersion results for the ballistic case and the rollstabilized case. Total maneuver-canard size was $18.6 \mathrm{~cm}^{2}$.

\section{Dispersion Simulations}

Dispersion simulations were performed to test control system robustness and effectiveness in eliminating error due to launch perturbations and atmospheric winds. For several different maneuver-canard sizes, 200 Monte Carlo simulations were performed. Each simulation incorporated sensor noise and bias errors, launch errors, and atmospheric winds. Uncontrolled circular error probable (CEP) was $113 \mathrm{~m}$ at a range of approximately $5500 \mathrm{~m}$. The nominal unperturbed initial conditions for all Monte Carlo simulations are the same as the unperturbed values given in Table 1 . Table 3 lists initial condition standard deviations and wind parameters, and Table 4 lists feedback signal noise and bias standard deviations. Note that the states $v, q$, and $r$ are not used in the control system and are therefore not included in Table 4 . Also note that noise and bias errors shown in Table 4 are commensurate with error levels associated with typical projectile state estimators. Wind direction is a uniform random variable between 0 and $2 \pi$.

Figure 15 shows ballistic dispersion results as well as an example of controlled dispersion results for the roll-stabilized mortar projectile using a total maneuver-canard area of $18.6 \mathrm{~cm}^{2}$. Notice that the control system effectively reduced the ballistic CEP of 113 to 13 m. Furthermore, Fig. 16 shows controlled CEP for various total maneuver-canard areas for both the roll-stabilized configuration and the rolling configuration. Note that the bank-to-turn design achieves significantly greater CEP reduction for a given canard size, reflecting the higher control authority inherent in its design.

\section{Trade Study: Maneuver Canard Placement}

A trade study was conducted to examine the effect of maneuvercanard placement along the projectile station line on control system performance. Typically, canard placement farther from the projectile mass center is desirable, since this leads to larger control moments and therefore increased control authority. This study demonstrates that the body pitching dynamics caused by large canard control

Table 3 Initial condition standard deviations

\begin{tabular}{lc}
\hline \hline States & Initial condition $\sigma$ \\
\hline$x, y, z$ & $0.0 \mathrm{~m}$ \\
$\phi$ & $0.0 \mathrm{deg}$ \\
$\theta$ & $0.69 \mathrm{deg}$ \\
$\psi$ & $0.40 \mathrm{deg}$ \\
$u$ & $4.72 \mathrm{~m} / \mathrm{s}$ \\
$v$ & $0.73 \mathrm{~m} / \mathrm{s}$ \\
$w$ & $0.73 \mathrm{~m} / \mathrm{s}$ \\
$p$ & $1.0 \mathrm{rad} / \mathrm{s}$ \\
$q$ & $1.0 \mathrm{rad} / \mathrm{s}$ \\
$r$ & $1.0 \mathrm{rad} / \mathrm{s}$ \\
Wind magnitude & $4.3 \mathrm{~m} / \mathrm{s}$ \\
\hline \hline
\end{tabular}

Table 4 Feedback signal noise and bias standard deviations

\begin{tabular}{ccc}
\hline \hline Feedback signal & Noise $\sigma$ & Bias $\sigma$ \\
\hline$x$ & $1.52 \mathrm{~m}$ & $0.30 \mathrm{~m}$ \\
$y$ & $1.52 \mathrm{~m}$ & $0.30 \mathrm{~m}$ \\
$z$ & $1.52 \mathrm{~m}$ & $0.91 \mathrm{~m}$ \\
$\phi$ & $0.06 \mathrm{deg}$ & $0.06 \mathrm{deg}$ \\
$\theta$ & $0.06 \mathrm{deg}$ & $0.06 \mathrm{deg}$ \\
$\psi$ & $0.06 \mathrm{deg}$ & $0.06 \mathrm{deg}$ \\
$u$ & $0.91 \mathrm{~m} / \mathrm{s}$ & $0.91 \mathrm{~m} / \mathrm{s}$ \\
$w$ & $0.91 \mathrm{~m} / \mathrm{s}$ & $0.91 \mathrm{~m} / \mathrm{s}$ \\
$p$ & $0.1 \mathrm{rad} / \mathrm{s}$ & $0.1 \mathrm{rad} / \mathrm{s}$ \\
\hline \hline
\end{tabular}




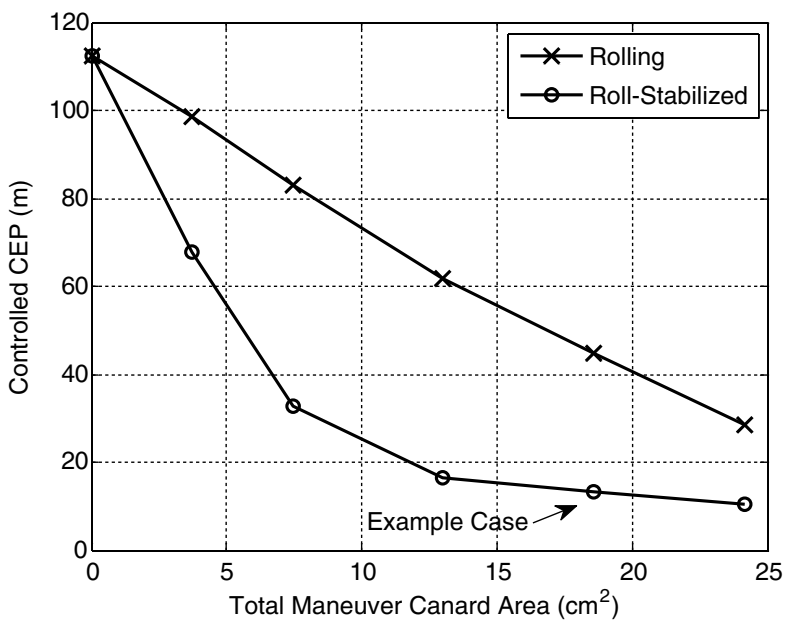

Fig. 16 Controlled CEP vs total maneuver-canard area.

moments must be taken into account during control law selection when using this fixed-angle canard configuration, since rapid changes in the error vector in the body-fixed frame can result in overactive roll control. The situation is exacerbated by the tendency of mortar projectiles to exhibit relatively large angular responses to control inputs due to the interference of the body with airflow over the stabilizing fins.

To examine the effect of canard placement, two trajectories are compared. The first, termed nominal placement, is the identical rollstabilized trajectory shown in the Example Trajectory section. Recall that maneuver canards are placed approximately $2.8 \mathrm{~cm}$ in front of the mass center. The second trajectory, termed forward placement, is identical to the nominal placement trajectory with the exception that canards are moved forward to $9.1 \mathrm{~cm}$ ahead of the mass center.

Figure 17 demonstrates that both controlled trajectories effectively eliminate trajectory errors in deflection. Range errors were also eliminated, with trajectory profiles very similar to those shown in the controlled traces in Figs. 5 and 6 . For the forward-placement case, maneuver-canard extension results in significantly higher angle of attack (Fig. 18), as expected. This causes the faster control response exhibited in Fig. 17. However, larger maneuver-canard moments result in higher pitch rates. As the projectile approaches the desired trajectory, large pitch oscillations cause rapid changes in the sign of the roll-angle error $\Delta \phi$, as shown in Fig. 19. This leads directly to overactive roll control activity, as can be clearly seen in the maneuver and roll-canard time histories shown, respectively, in Figs. 20 and 21. In this manner, maneuver control response directly affects roll control performance (maneuver-canard response is shown for reference in Fig. 20). Specifically, due to the fixed-angle design of the

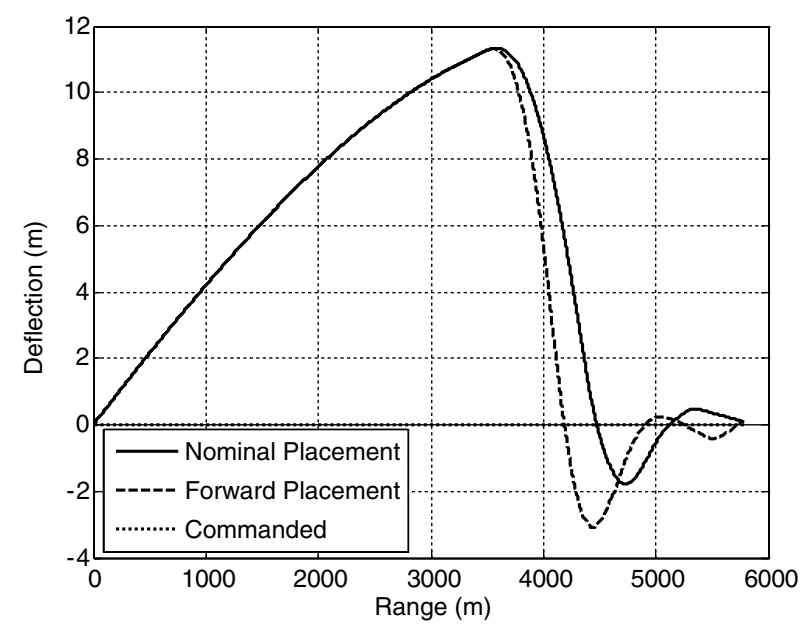

Fig. 17 Deflection vs range.
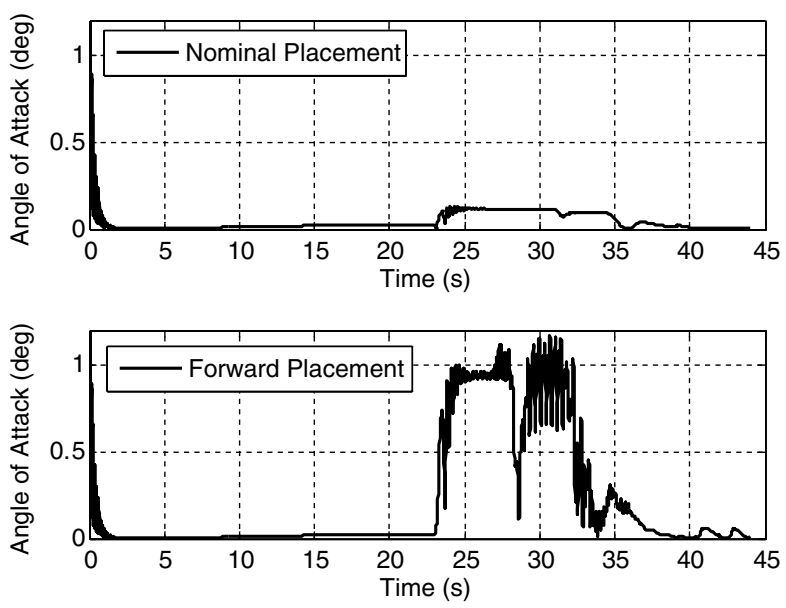

Fig. 18 Angle of attack vs time.
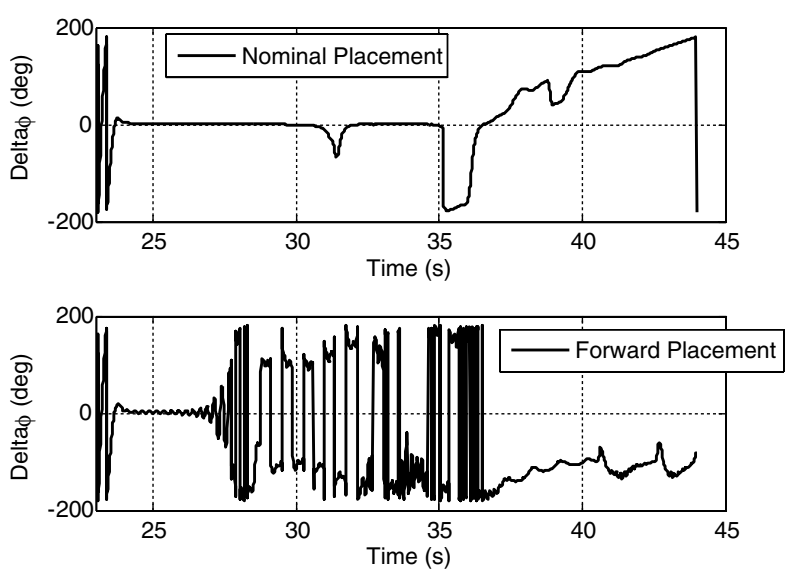

Fig. $19 \Delta \phi$ vs time.
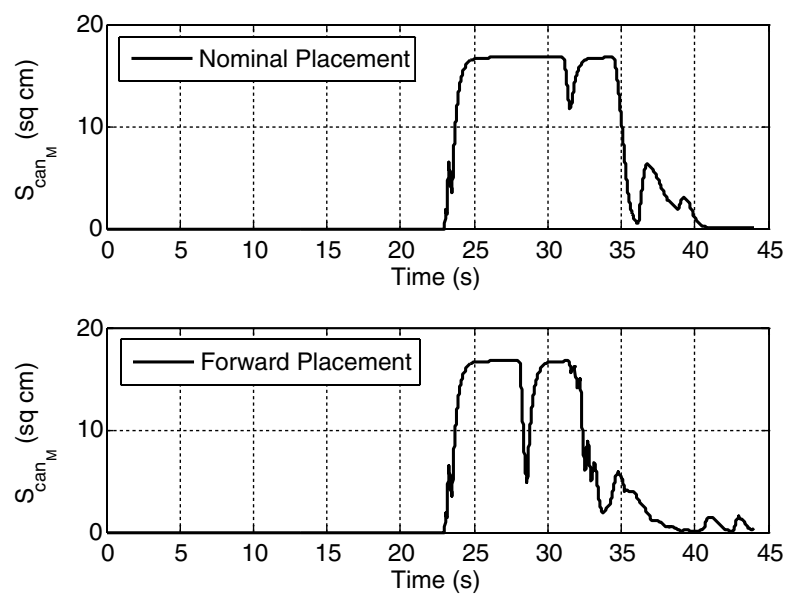

Fig. 20 Maneuver-canard area-time histories.

roll-stabilization canards, this bank-to-turn configuration is susceptible to overactive roll control when using control schemes based on errors in a body-fixed frame. The use of low-pass filtering techniques to mitigate this high-frequency response would result in delayed roll control response and reduced overall performance. Therefore, if maneuver canards are placed such that canard moments are large, the control law should account for this coupling of roll and maneuver control response and use error signals in a velocity-based frame rather than a body-fixed frame. 

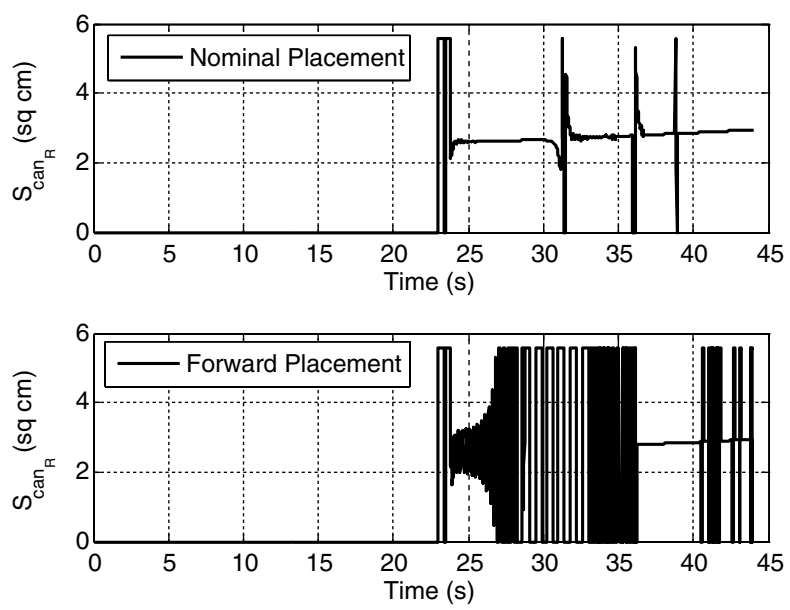

Fig. 21 Roll-canard area-time histories.

\section{Conclusions}

A unique design of a bank-to-turn roll-stabilized mortar projectile is proposed. The projectile uses two sets of reciprocating canards for roll and maneuver control. Through the use of example simulations and trade studies, it is shown that the roll-stabilized design is effective at significantly reducing dispersion error in the presence of launch disturbances, atmospheric winds, and sensor errors. Furthermore, it is shown that the roll-stabilized system exhibits higher control authority and desirable flight characteristics compared with similar rolling configurations. In addition, low-bandwidth canard actuators can be used, reducing the cost and complexity of the control system. Finally, trade studies show that large maneuver-canard moments can cause coupling between the maneuver and roll control system loops. Overall, the roll-stabilized design presented here reduces the burden on sensor packages, allows for the use of lower-cost actuators, reduces drag compared with dithering canard designs, and exhibits better control performance than comparable rolling reciprocating canard designs.

\section{References}

[1] Fink, R. D., "The USAF Stability and Control DATCOM," Air Force Wright Aeronautical Labs., TR-83-3048, Wright-Patterson AFB, OH, April 1978.

[2] Smith, J. A., Smith, K. A., and Topliffe, R., "Feasibility Study for Application of Modular Guidance and Control Units to Existing ICM Projectiles," U.S. Army Armament Research and Development Command, ARLCD-CR-79001, Nov. 1978.

[3] Costello, M., "Extended Range of a Gun Launched Smart Projectile Using Controllable Canards," Shock and Vibration, Vol. 8, Nos. 3-4, 2001, pp. 203-213.

[4] Morrison, P. H., "A Lesson Learned About Cannon-Launched Guided Projectiles," Journal of Guidance and Control, Vol. 3, No. 2, MarchApril 1980, pp. 154-157. doi:10.2514/3.55963

[5] Morrison, P. H., and Amberntson, D. S., "Guidance and Control of a Cannon-Launched Guided Projectile," Journal of Spacecraft and Rockets, Vol. 14, No. 6, June 1977, pp. 328-334. doi: $10.2514 / 3.57205$

[6] Yager, J. K., and Froysland, J. L., "Improving the Effects of Fires with Precision Munitions," Field Artillery, March-April 1997, pp. 5-7.

[7] Tang, L., Liu, D. D., and Chen, P. C., "Extension of Projectile Range Using Oblique-Wing Concept," Journal of Aircraft, Vol. 44, No. 3, May-June 2007, pp. 774-779. doi: $10.2514 / 1.21194$

[8] Blosser, O. K., "Naval Surface Fires and the Land Battle," Field Artillery, Sept.-Oct. 1996, pp. 41-45.

[9] Grubb, N. D., and Belcher, M. W., "Excalibur: New Precision Engagement Asset in the Warfight," Fires, Oct.-Dec. 2008, pp. 14-15.

[10] Moorhead, J. S., "Precision Guidance Kits (PGKs): Improving the Accuracy of Conventional Cannon Rounds," Field Artillery, Jan.Feb. 2007, pp. 31-33.

[11] Etkin, B., Dynamics of Atmospheric Flight, Wiley, New York, 1996, pp. 103-104.

[12] Murphy, C., "Free Flight of Symmetric Missiles," Ballistics Research Lab. Rept.BRL-1216, Aberdeen Proving Ground, MD, 1963.

[13] Costello, M., and Anderson, D., "Effect of Internal Mass Unbalance on the Stability and Terminal Accuracy of a Field Artillery Projectile," 1996 AIAA Atmospheric Flight Mechanics Conference, San Diego, CA, AIAA Paper 1996-3447, 1996.

[14] "Prodas Version 3 Documentation," Arrow Tech Associates, Burlington, VT, 2000. 